\title{
WAVELET DECOMPOSITION OF SUBMILLIMETER SOLAR RADIO BURSTS
}

\author{
V. S. MAKHMUTOV*, J.-P. RAULIN, C. G. GIMÉNEZ DE CASTRO, \\ P. KAUFMANN** and E. CORREIA*** \\ CRAAM, Universidade Presbiteriana Mackenzie, Rua da Consolação 896, \\ 01302-000 São Paulo, SP, Brazil
}

(Received 21 April 2003; accepted 6 October 2003)

\begin{abstract}
We present the results of wavelet decomposition of fast-time structures during four solar flares observed by the Solar Submillimeter-wave Telescope at $212 \mathrm{GHz}$. The result of the analysis shows (1) observational evidence on the existence of submm-emission time variations in the range from a few tens of millisecond up to few seconds during solar flares, and (2) that when a solar flare is in progress the time scales reduce as the bulk of the emission flux time variations is increasing.
\end{abstract}

\section{Introduction}

The analysis of the time variations of flare emissions in radio, X-ray, $\gamma$-ray, and white-light energy ranges allows us to study the primary energy release and transport processes at the flaring site. Over the last decades sub-second time structures have been found and investigated at microwaves and millimeter flare emission (Kaufmann et al., 1980, 1985, 2000, 2001a; Takakura et al., 1983; Correia and Kaufmann, 1987; Makhmutov et al., 1998; Raulin et al., 1998; Giménez de Castro et al., 2001), as well as X-ray emission from the solar observations obtained by SMM/HXRBS (Kiplinger et al., 1983), CGRO/BATSE (Machado et al., 1993; Aschwanden et al., 1998), GRANAT/PHEBUS (Vilmer et al., 1994), etc. Significant correlation between time structures at microwave, millimeter and hard X-ray, $\gamma$-ray domains were found (Takakura et al., 1983; Kaufmann et al., 1985, 2001b). Nevertheless, it has been stressed that 'there is a need for still more detailed studies of hierarchic burst time structures based on extended nonlinear time series analyses...' (Krüger et al., 1994).

In 1999 the Solar Submillimeter Telescope (SST) started solar observations at 212 and $405 \mathrm{GHz}$ at El Leoncito site (Argentinean Andes, altitude of $2550 \mathrm{~m}$ ) (Kaufmann et al., 2001a) with four beams at $212 \mathrm{GHz}\left(\mathrm{HPBW}=4^{\prime}\right)$ and two beams at $405 \mathrm{GHz}\left(\mathrm{HPBW}=2^{\prime}\right)$. The SST high-sensitivity and high-time-resolution data

*Permanent position at Lebedev Physical Institute RAS, Leninsky prospekt, 53, 119991 Moscow, Russia.

**Part time at CSS, UNICAMP, SP, Brazil.

***Agreement with INPE, São Josè dos Campos, SP, Brazil.

Solar Physics 218: 211-220, 2003.

(c) 2003 Kluwer Academic Publishers. Printed in the Netherlands. 
(1 ms) allow one to study the solar radio emission flux variations at various time scales (from a few ms to few minutes).

The first results on the observations of the rapid subsecond submm-pulses during solar flares were reported by Kaufmann et al. (2001b, 2002). The goal of this short paper is to present observational results on the time evolution properties of submm-time structures during solar flares using a wavelet decomposition technique.

\section{Observational Data and Their Analysis}

We selected four solar flares recorded at $212 \mathrm{GHz}$ by the SST in 2001: 6 April, 25 August, 28 November, and 13 December. Using a multiple-beam technique (Giménez de Castro et al., 1999) we determined the solar flux variations at $212 \mathrm{GHz}$ during the events (with the exception of the 13 December event). We do not use the $405 \mathrm{GHz}$ data since only one event was recorded at this frequency.

\subsection{METHOD}

A multi-resolution analysis based on wavelet transform is used to define time structures and the time evolution of their characteristics during solar flares. Similar studies at different frequencies and energy domains were presented in the past, (e.g., Kurths and Schwarz, 1994; Aschwanden et al., 1998; Giménez de Castro et al., 2001). In our analysis we used the technique described in Giménez de Castro et al. (2001) based on the multi-resolution method proposed by Mallat (1989) and the algorithm developed in Bendjoya, Petit, and Spahn (1993).

The observed emission-flux time variation $F(t)$ can be expressed in the form

$$
F(t)=\sum_{j} \sum_{k} d_{j, k} w_{j, k}(t),
$$

where $d_{j, k}$ are the wavelet coefficients and $w_{j, k}$ are the wavelet basis functions evaluated for each time scale from a triangle 'mother wavelet'. We present the results of application of the multi-resolution analysis to the data in terms of scalograms $P(t, T)$ and scalegrams $S(T)$ corrected by the noise level (e.g., Kurths and Schwarz, 1994; Aschwanden et al., 1998; Giménez de Castro et al., 2001). Scalograms show for each time scale $T$ (or $T S$ ) the wavelet coefficient $\left(d_{j, k}\right)$ changes in time, $t$. Scalegrams represent in some way a Fourier power spectral density $P(v=1 / T)$ as a function of the frequency $v$, averaged during the time interval selected. The power-law slope $(\beta)$ of the given scalegram can be calculated (e.g., Aschwanden et al., 1998). To exclude a noise level of the data from the chosen scalegrams we have evaluated a noise scalegram with a significance level of 99\% level using a Monte-Carlo simulation of 100000 pseudo-random time series containing 32000 points (for each of the series; for details see Giménez 
de Castro et al., 2001). In the scalegrams plots we present a noise scalegram estimated from preflare measurements and scalegrams during the events with the subtraction of the noise level as it was proposed by Scargle et al. (1993), i.e., $S(T)=S_{\text {event }}(T)-S_{\text {noise }}(T)$.

The details of submillimeter instrumentation in relation to the r.m.s. noise fluctuations of the SST and possible system noise temperature contributions to the result of wavelet decomposition of fast time structures are presented in Raulin et al. (2003a). Here we only note that the system noise temperature $T_{\text {sys }}$ accounts for the temperature of the receivers $T_{\text {rec }}$, and for all temperatures of sources located along the line of sight: the sky, the quiet Sun, and eventually a localized solar burst. The level of r.m.s. noise fluctuations can be determined from the well-known equation

$$
\Delta T=\frac{T_{\text {sys }}}{\sqrt{t \Delta f}},
$$

where $T_{\text {sys }}$ is the system noise temperature, $\Delta f$ is the bandwidth of the receivers ( $\Delta f=1 \mathrm{GHz}$ ), and the time constant is $t$. The noise r.m.s. fluctuations, due to the different contributions to the system noise temperature, have been estimated during four solar events under consideration. As the result, we found that $\Delta T$ increased by a factor $\leq 2$ due to the flare emission. This means that the flare emission at $212 \mathrm{GHz}$ did not produce any significant increase of the r.m.s. noise fluctuation level compared to the flux (temperature) fluctuations due to solar burst pulsations, as will be shown later.

\subsection{SOLAR FlARE OBSERVATIONS}

The solar flare observations at $212 \mathrm{GHz}$ are presented in Figures 1-4, where the upper panels (a) show the flux density (or antenna temperature) time profile. The results of wavelet transform of these time profiles, in terms of scalograms and scalegrams, are presented in the panels (b) and (c) of the figures. The bottom panels (d) and (e) show the $212 \mathrm{GHz}$ flare emission time profile during sample intervals of $1 \mathrm{~s}$ (or $3 \mathrm{~s}$ ) close to the main phase of the solar bursts. Panels (e) show the corresponding parts of scalograms during these intervals. The 3 times the r.m.s. noise fluctuations level $(3 \Delta T)$ are shown by a vertical thick bar at the left in panels (d).

6 April 2001 X5.6 class solar flare occurred in the active region NOAA 9415 during 19:10-20:29 UT. The comparison of submm-pulses and $\gamma$-rays has been presented in Kaufmann et al. (2002). Figure 1 presents the $212 \mathrm{GHz}$ solar flux time profile during the event (upper panel; integration time of the data is $10 \mathrm{~ms}$ for all events). The gaps in the flare data are due to calibration and opacity measurements. Panel (b) shows the scalogram contours. It is seen that close to the peak of the event the shortest time scales of flux variations appeared. To compare the characteristics of subsecond time scales through the flare development we selected nine time intervals with 3-s duration (shown by vertical arrows in Figure 1 top) for 
which we present the 3-s averaged scalegrams panel (c). We define a minimum time scale $\left(T_{\min }\right)$, with a $99 \%$ confidence level, by the intersection of a noise scalegram (thick line) and a given scalegram for each 3-s interval throughout the event (thin lines). Examination of the scalegrams in the panels (c) shows (1) time scales (TS) are $\sim 330 \mathrm{~ms}$ at the beginning of the event (line 1), then they decrease to about $\sim 160 \mathrm{~ms}$ (line 3,4) and became shorter than $100 \mathrm{~ms}$ close to the peak of the event (panels (c) and (e)). The power-law slope $(\beta)$ of the maximum scalegram (line 5) is 2.2. During the decay phase of the event the time scale of the variations becomes more prolonged, like that obtained in the ascending phase of the event; (2) the power of all time scales (TS $\leq 1 \mathrm{~s}$ ) is increasing when the flux density increases up to the peak emission.

Similar analyses were done for other 3 events.

25 August 2001 solar flare. The X5.3 class solar flare occurred in NOAA 9591 during 16:23-17:04 UT. It showed strong high-energy X-ray and $\gamma$-ray signal $(E \geq 10 \mathrm{MeV})$ as well as strong micro-, submm-wave emissions. A more detailed analysis of this event in relation to the physical processes observed at the flare will be presented in Raulin et al. (2003b).

Figure 2 shows the appearance of the fastest-time-scale flux variations close to the maximum of the event (panels (b-e)). They become shorter than $20 \mathrm{~ms}$ at about 16:31 UT. Furthermore, the power of all time scales increased significantly, e.g., the power of $125 \mathrm{~ms}$ variations changes by a factor $\sim 400$ in 40 seconds (line 3 and 5 in panel (c)). For the maximum scalegram $\beta=2.0$ (line 5).

28 November 2001 solar flare classified as M6.9 class occurred in NOAA 9715 between 16:26 and 16:41 UT. The duration of this event is about $110 \mathrm{~s}$ at $212 \mathrm{GHz}$ and the peak flux value was $\sim 150$ s.f.u.

Figure 3 shows the time profiles of $212 \mathrm{GHz}$ emissions and seven intervals of 3 seconds which were selected to analyse the temporal evolution of the time scales during the event (upper panel). The time-scale evolution tendency as seen in the scalegrams (panel (c) and (e)), shows that closest to the peak of the event the shortest time scales of flux variations are detected.

The minimum time scale at $212 \mathrm{GHz}$ emission during the event maximum is about of $50 \mathrm{~ms}$ (line 3 in panel (c)). The power-law slope of the scalegrams was in the range from 1.0 to 1.8 during the event. Around the maximum of the event the scalegrams are characterized by $\beta=1.8$ (line 3 in panel (c)).

13 December 2001 X6.2 solar flare occurred in NOAA 9733 between 14:2314:49 UT. Contrary to the previous events, the flare emission at $212 \mathrm{GHz}$ was less intense and does not show clear bulk (min) emission. However we clearly see the appearance of fast time structures from the original $212 \mathrm{GHz}$ antenna temperature records. Figure 4 shows the antenna temperature time profile as recorded by beam 4 of the SST antenna (upper panel). The scalograms obtained during the event are shown in the panel (c) and (e). The evolution of time scales shows the fastest emission variation during the time interval 14:28-14:30 UT, which is close to the maximum of the event as observed by GOES-10. There is a clear indication of the 

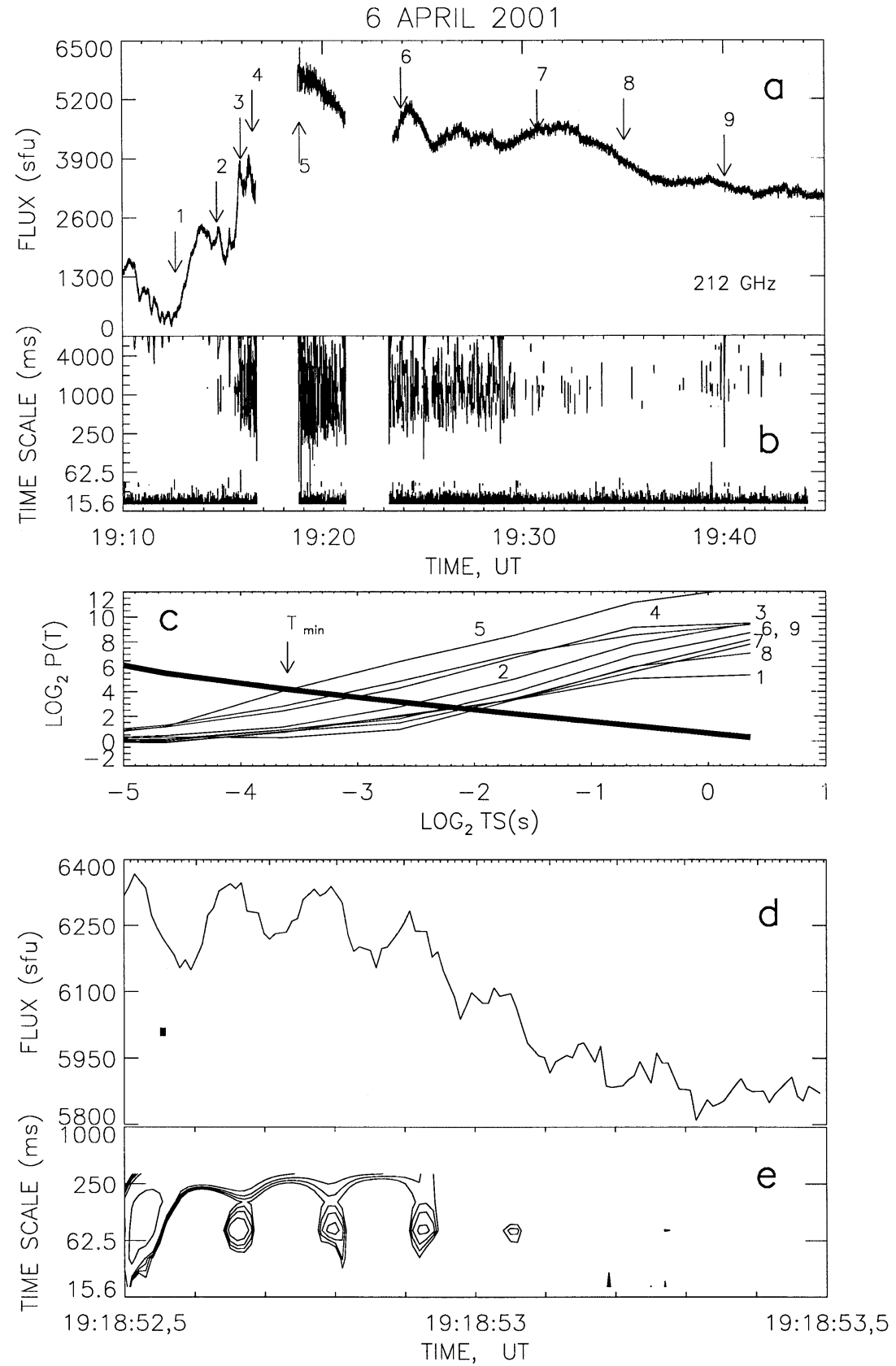

Figure 1. (a) The SST $212 \mathrm{GHz}$ flux time profile during solar flare on 6 April 2001. Vertical arrows indicate the 3-s time intervals (from 1 to 9) of the data for which time-averaged scalegrams are shown in panel (c). (b) The scalograms evaluated from 10-ms SST data during the event. (c) The 3-seconds averaged scalegrams obtained during the selected intervals (1-9). The thick line is a 'noise' level scalogram. Above this line the confidence level of time scales is $99 \%$, i.e., they are significant. The $212 \mathrm{GHz}$ flare-emission time profile during sample interval of $1 \mathrm{~s} \mathrm{((d)} \mathrm{time} \mathrm{constant} \mathrm{is} 10 \mathrm{~ms})$ and corresponding part of scalograms (e) Vertical thick bar on the left in (d) corresponds to 3 times the r.m.s. noise fluctuations level. 

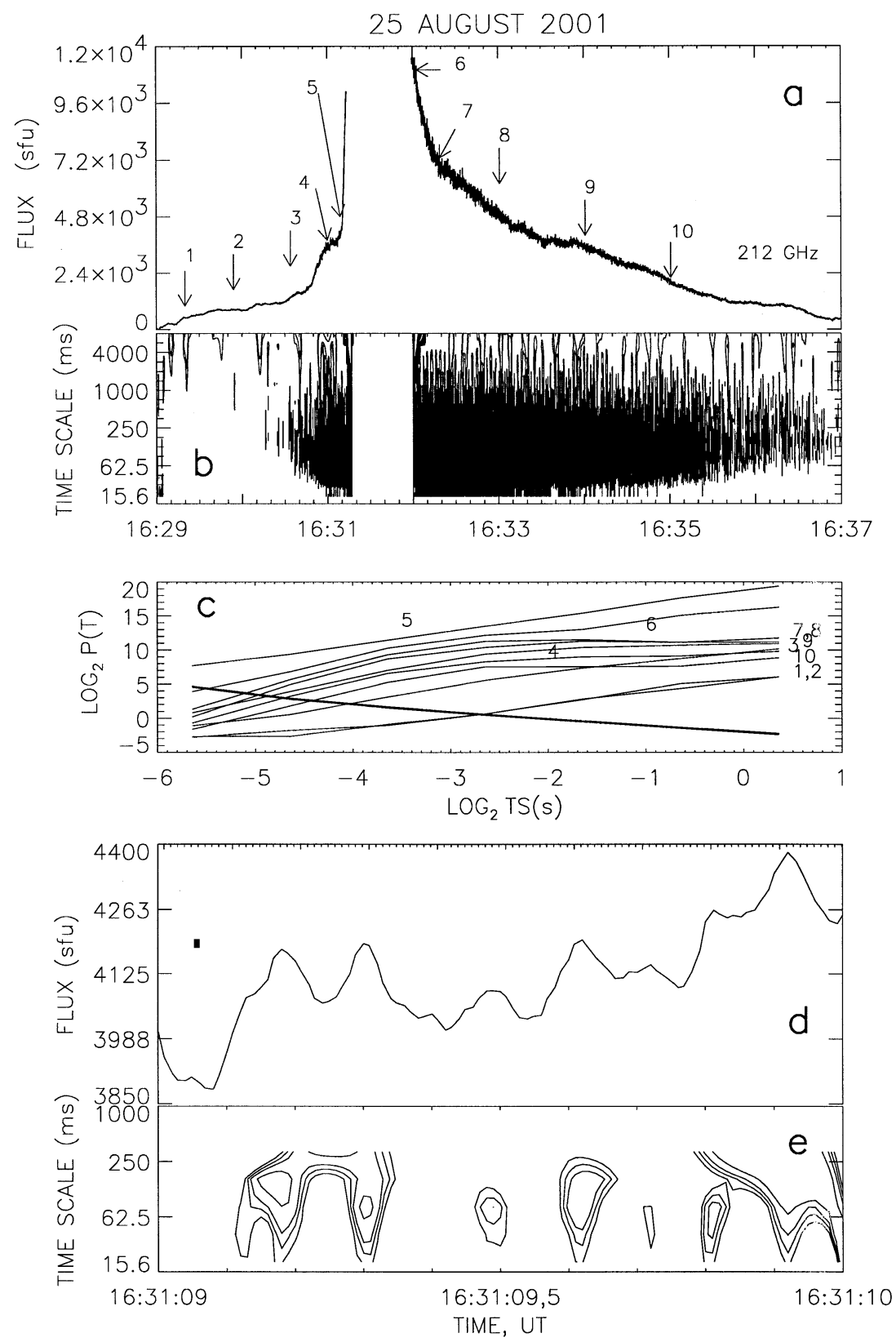

Figure 2. Same as in Figure 1 but for the solar flare on 25 August 2001. 

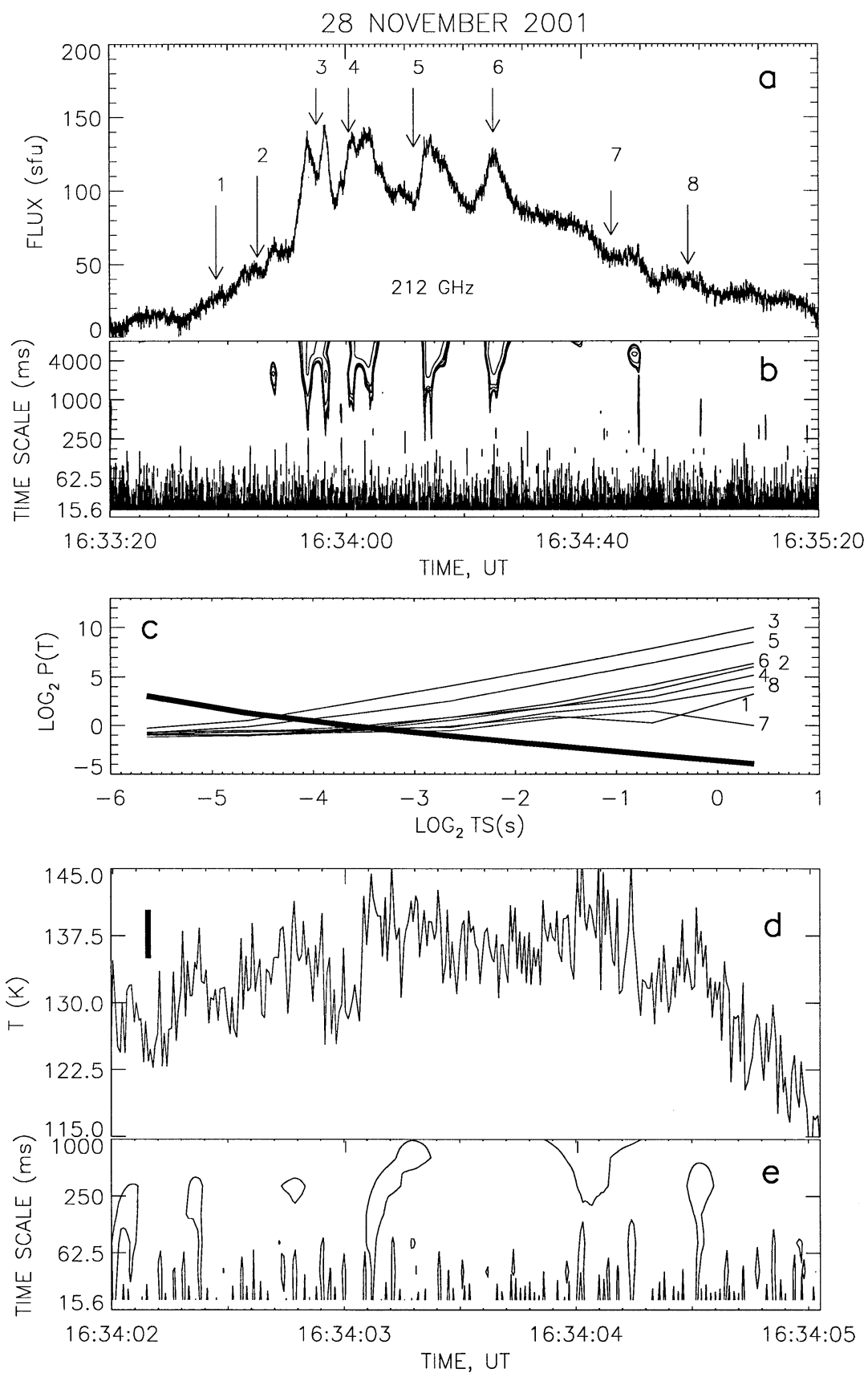

Figure 3. Same as in Figure 1 but for the solar flare on 28 November 2001. 

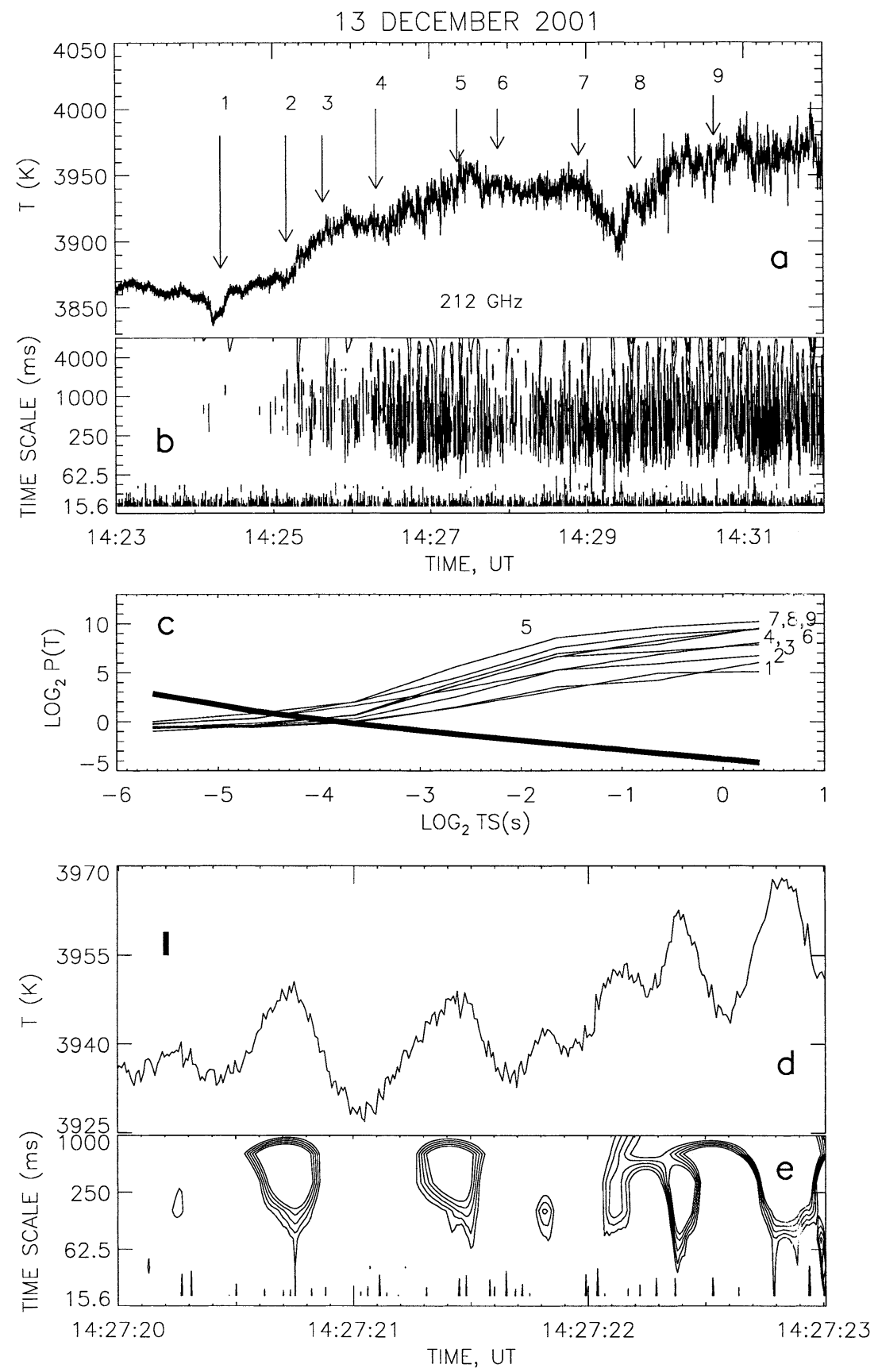

Figure 4. Same as in Figure 1 but for the solar flare on 13 December 2001. The upper panel shows the antenna temperature time profile during the event. 
existence of time scales down to $50 \mathrm{~ms}$ in submm emission (panel (c) and (e)). The maximum $\beta$ value during the event is equal to 2.2 .

\section{Conclusions}

We presented the results of the analysis of observations of four solar flares on 6 April 2001, 25 August 2001, 28 November 2001, and 13 December 2001 provided by the multiple-beam Solar Submillimeter Telescope at $212 \mathrm{GHz}$. We used a multiresolution time analysis based on wavelet transform to define the solar radioemission time structures and their evolution during the events. All four examples have shown (1) observational evidence of the existence of subsecond time scales at $212 \mathrm{GHz}$; (2) the evolution of the subsecond time structures during the flare shows that the time scale decreases down to $20-100$ ms close to the peak of the event; (3) the power of all time scales $(\leq 1 \mathrm{~s})$ significantly increases around the maximum of the event and (4) the power-law slope $(\beta)$ of the scalegrams around solar flare maximum changes in the range $1.8-2.2$. This is in accordance with the estimations of $\beta$ during strong impulsive X-ray flares (Aschwanden et al., 1998) as well as that obtained at $37 \mathrm{GHz}$ (Schwarz et al., 1998) and $48 \mathrm{GHz}$ (Giménez de Castro et al., 2001) during the main phase of solar flares. The detailed analysis of the origin of these fast time structures is out of the scope of this paper. Nevertheless, we note: (1) the 25 August 2001 solar flare shows that the more energetic and very fast increase of solar radio-, X-ray, and $\gamma$-ray emissions were related in time to the appearance of very compact sources $\left(5^{\prime \prime}-7^{\prime \prime}\right)$ in soft X-rays according to Yohkoh/SXT and TRACE observations (Raulin et al., 2003b). At this moment the time scales of $212 \mathrm{GHz}$ emission are the shortest (see the scalegram 5 in panel (c) of Figure 2). Assuming a very simple relation between $T_{\min }$ and the loop size $r$ in the form $T_{\min }(\mathrm{s}) \approx 0.5 r(\mathrm{~cm}) \times 10^{-9}$ (Aschwanden et al., 1998), with $r \approx 6 \times 10^{7} \mathrm{~cm}$ we get $T_{\min } \approx 20 \mathrm{~ms}$; (2) the fast time structures observed at microwave, submm-wave emission might be consistent with the coalescence model of flare energy release (Pritchett and Wu, 1979; Tajima et al., 1987, etc.) assuming a small size for the energy release region $\left(l \leq 10^{7-8} \mathrm{~cm}\right)$.

\section{Acknowledgements}

SST is operated with partial support from agencies, FAPESP, Brazil, grant No. 99/06126-7, CNPq, Brazil, grant No. 69.0190/02-6 and CONICET, Argentina. VSM acknowledges financial support of CNPq through grant No. 300607/01-6(NV). JPR thanks to CNPq for the contract No. 300782/96-9. The authors sincerely appreciate the useful comments of the referee.

\section{References}

Aschwanden, M. J., Kliem, B., Schwarz, U., Kurths, J., Dennis, B. R., and Schwartz, R. A.: 1998, Astrophys. J. 505, 941. 
Bendjoya, Ph., Petit, J.-M., and Spahn, F.: 1993, ICARUS 105, 385.

Correia, E. and Kaufmann, P.: 1987, Solar Phys. 111, 143.

Giménez de Castro, C. G., Raulin, J.-P., Makhmutov, V. S., Kaufmann, P., and Costa, J. E. R.: 1999, Astron. Astrophys. Suppl. 140, 373.

Giménez de Castro, C. G., Raulin, J.-P., Mandrini, C. H., Kaufmann, P., and Magun, A.: 2001, Astron. Astrophys. 366, 317.

Kaufmann, P.: 2002, Solar Observations at Submillimeter Wavelengths, Lecture Notes in Physics Series, Springer-Verlag, Berlin.

Kaufmann, P., Strauss, F. M., Opher, R., and Laporte, C.: 1980, Astron. Astrophys. 87, 58.

Kaufmann, P., Correia, E., Costa, J. E. R., Zodi Vaz, A. M., and Dennis, B. R.: 1985, Nature 313, 380.

Kaufmann, P., Trottet, G., Giménez de Castro, C. G., Costa, J. E. R., Raulin, J.-P., Schwartz, R. A., and Magun, A.: 2000, Solar Phys. 197, 361.

Kaufmann, P., Costa, J. E. R., Giménez de Castro, C. G., Hadako, Y. S., Kingsley, J. S., Kingsley, R. K., Levato, H., Marun, A., Raulin, J.-P., Rovira, M., Correia, E., and Silva, A. V. R.: 2001a, Proc.SBMO/IEEE MTT - S International Microwave and Optoelectronics Conference, August 6 -10, 2001, Belém, Para, Brazil, reprinted in Telecomunicasões 4, 18.

Kaufmann, P., Raulin, J.-P., Correia, E., Costa, J. E. R., Giménez de Castro, C. G., Silva, A. V. R., Levato, H., Rovira, M., Mandrini, C., Fernandez-Borda, R., and Bauer, O. H.: 2001b, Astrophys. J. 548, L95.

Kaufmann, P., Raulin, J.-P., Melo, A. M., Correia, E., Costa, J. E. R., Giménez de Castro, C. G., Silva, A. V. R., Yoshimori, M., Hudson, H. S., Gan, W. Q., Gary, D. E., Gallaher, P. T., Levato, H., Marun, A., and Rovira, M.: 2002, Astrophys. J. 574, 1059.

Kiplinger, A. L., Dennis, B. R., Emslie, A. G., Frost, K. J., and Orwig, L. E.: 1983, Astrophys. J. 265, L99.

Krüger, A. , Kliem, B., Hildebrandt, J., and Zaitsev, V. V.: 1994, Astrophys. J. Suppl. Ser. 90, 683.

Kurths, J. and Schwarz, U.: 1994, Space Sci. Rev. 68, 171.

Machado, M. E., Oug, K. K., Emslie, A. G., Fishman, G. J., Meegan, C., Wilson, R., and Paciesas, W. S.: 1993, Adv. Space Res. 13, 175.

Makhmutov, V. S., Costa, J. E. R., Raulin, J.-P., Kaufmann, P., Lagrotta, P. R., Giménez de Castro, C. G., Magun, A., and Arzner, K.: 1998, Solar Phys. 178, 393.

Mallat, S. G.: 1989, IEEE Transactions on Pattern Analysis and Machine Intelligence 11, 674.

Pritchett, P. L. and Wu, C. C.: 1979, Phys. Fluids 22, 2140.

Raulin, J.-P., Kaufmann, P., Olivieri, R., Correia, E., Makhmutov, V. S., and Magun, A.: 1998, Astrophys. J. 498, L173.

Raulin, J.-P., Kaufmann, P., Giménez de Castro, C. G., Pacini, A. A., Makhmutov, V. S., Levato, H., and Rovira, M.: 2003a, Astrophys. J. 592, 580.

Raulin, J.-P., Makhmutov, V. S., Kaufmann, P., Luethi, T., Hudson, H. S., Gary, D. E., and Yoshimori, M.: 2003b, Astrophys. J., in preparation.

Scargle, J. D., Steiman-Cameron, T., Joung, K., Donoho, D. L., Crutchfield, J. P., and Imamura, J.: 1993, Astrophys. J. 411, L91.

Schwarz, U., Kurths, J., Kliem, B., Krüger, A., and Urpo, S. N.: 1998, Astron. Astrophys. Suppl. Ser. 127, 309.

Tajima, T., Sakai, J., Nakajima, T., Kosugi, T., Brunel, F., and Kundu, M. R.: 1987, Astrophys. J. 321, 1031.

Takakura, T., Kaufmann, P., Costa, J. E. R., Degaonkar, S. S., Ohki, K., and Nitta, N.: 1983, Nature 302, 317 .

Vilmer, N., Trottet, G., Barat, C., Dezalay, J. P., Talon, R., Sunyaev, R., Terekhov, O., and Kuznetsov, A.: 1994, Space Sci. Rev. 68, 233. 